\title{
IMPACTO DA FISIOTERAPIA NA CAPACIDADE FUNCIONAL E QUALIDADE DE VIDA DE INDIVÍDUOS COM A SÍNDROME PÓS-POLIOMIELITE
}

\author{
Ana Carolina de Lima Calado¹, Francisco de Assis Coutinho Pontes \\ Júnior², Karen Lúcia de Freitas Moreira ${ }^{3}$, Stênio Melo Lins da Costa ${ }^{3}$, \\ Maria Eduarda Camilo Damião ${ }^{3}$, Isolda Maria Barros Torquato ${ }^{3}$
}

\footnotetext{
${ }^{1}$ Fisioterapeuta, Universidade Federal da Paraíba, João Pessoa-PB, Brasil. ${ }^{2}$ Fisioterapeuta, Hospital Universitário Lauro Wanderley, João Pessoa, PB, Brasil. ${ }^{3}$ Fisioterapeuta, Departamento de Fisioterapia, Universidade Federal da Paraíba, João Pessoa, PB, Brasil.

E-mail para correspondência: isoldaufcg@gmail.com
}

\begin{abstract}
Resumo
A Síndrome Pós-Poliomite se caracterizada pela presença de sintomas como fadiga, atrofia, fraqueza muscular e dor mioarticular resultante de nova deterioração neuronal motora anos após a forma aguda da doença, ou seja, a poliomielite. Objetivou-se analisar o impacto do tratamento fisioterapêutico em grupo na melhora da dor, capacidade funcional e qualidade de vida de indivíduos com a Síndrome Pós-Pólio. Pesquisa transversal realizada com 10 pacientes usuários da Fundação Centro Integrado de Apoio ao Portador de Deficiência de João Pessoa, PB. Realizou-se entrevista individual norteada por instrumentos objetivos para avaliação das variáveis dor (Escala Visual Analógica da Dor), capacidade funcional (Escala de Atividades de Vida Diária de Barthel) e qualidade de vida (WHOQOL-bref). A análise dos dados foi realizada por meio de estatística descritiva. O estudo foi aprovado pelo comitê de ética sob parecer 4.003.241. Resultados: Cerca de 80,0\% dos participantes apresentaram melhora do quadro álgico de grave para leve; da capacidade funcional de moderada para leve $(80,0 \%)$ e da qualidade de vida nos domínios físico, psicológico e relações sociais. Conclusão: os atendimentos fisioterapêuticos em grupo constituíram-se como uma estratégia efetiva na melhora da dor, capacidade funcional e qualidade de vida de indivíduos com a SPP.

Palavras-chave: Dor; Fisioterapia; Funcionalidade; Qualidade de vida.
\end{abstract}

\begin{abstract}
Post-Polio Syndrome is characterized by the presence of symptoms such as fatigue, atrophy, muscle weakness and myoarticular pain resulting from a new motor neuronal deterioration years after the acute form of the disease,
\end{abstract}


that is, polio. The objective was to analyze the impact of group physical therapy treatment in improving pain, functional capacity and quality of life of individuals with Post-Polio Syndrome. Cross-sectional survey carried out with 10 patient users of the João Pessoa, PB, Integrated Center for the Support of the Disabled. Individual interviews were conducted, guided by objective instruments to assess the variables pain (Visual Analog Pain Scale), functional capacity (Barthel's Daily Activities Scale) and quality of life (WHOQOL-bref). Data analysis was performed using descriptive statistics. The study was approved by the research ethics committee of the number 4.003.241. Results: Approximately $80.0 \%$ of the participants showed improvement from severe to mild pain; functional capacity from moderate to light $(80.0 \%)$ and quality of life in the physical, psychological and social relations domains. Conclusion: group physiotherapy visits constituted an effective strategy to improve pain, functional capacity and quality of life of individuals with SPP.

Keywords: Pain; Physiotherapy; Functionality; Quality of life.

\section{Introdução}

A Poliomielite também conhecida como paralisia infantil, é uma doença infectocontagiosa aguda causada pelo poliovírus, composto por cadeia simples de ácido ribonucleico, que afeta os neurônios motores provocando fraqueza ou paralisia muscular (FARIA, 2015).

No final da década 80, estima-se que a doença foi responsável por acometer cerca de mil crianças diariamente. Nesse sentido, diante da gravidade epidemiológica a Organização Mundial da Saúde (OMS) estabeleceu metas para a erradicação da poliomielite até o ano 2000 (DURANTE; POZ, 2014).

Os casos de poliovírus selvagem diminuíram em mais de $99 \%$ desde 1988, quando foram estimados 350 mil casos em mais de 125 países endêmicos (OPAS, 2019). No Brasil, a última notificação de infecção pelo poliovírus selvagem ocorreu em 1989, na cidade de Souza/PB (BRASIL, 2018). A estratégia adotada para a eliminação do vírus foi centrada na realização de campanhas de vacinação, sendo a única maneira de prevenção da poliomielite (TAVARES, 2015).

Entretanto, na última década, apesar da erradicação da doença em alguns países, a atenção está voltada ao reaparecimento de sintomas semelhantes aos da poliomielite que acontecem cerca de 40-50 anos após a fase aguda da doença (ALMEIDA et al., 2016). A sintomatologia ocorre repentinamente e caracteriza-se, principalmente, por fadiga, atrofia, nova fraqueza muscular e dor mioarticular resultante de nova deterioração neuronal 
motora anos após a forma aguda da doença. A esse novo aparecimento do quadro clínico foi denominado como Síndrome Pós-Poliomielite ou Síndrome Pós-Pólio (SPP) (VENTURA, 2015).

Essa nova condição compreende um conjunto específico de problemas de saúde originados pelo vírus da pólio e que vem afetando pessoas acometidas pela doença décadas atrás. No final dos anos 70 , os sobreviventes começaram a apresentar sintomas semelhantes aos da fase aguda da poliomielite, resultando na diminuição da capacidade funcional e/ou no surgimento de novas incapacidades (SOARES et al., 2019). Estima-se que a prevalência da SPP mundial é de 22 a $87 \%$ entre as pessoas com sequela de poliomielite paralítica (RECHENCHOSKI et al., 2015).

Sua etiologia ainda é incerta, mas acredita-se que esteja relacionada a diversas teorias que focam alterações degenerativas ou disfunção da unidade motora sobrevivente devido à alta demanda metabólica ou do envelhecimento prematuro; o desuso ou uso excessivo da musculatura; a predisposição para a degeneração do neurônio motor devido às alterações gliais, vasculares e linfáticas causadas pela poliomielite aguda; a reativação viral; a síndrome imunomediada; e o efeito combinado do uso excessivo da musculatura, dor, aumento de peso ou outras doenças (VENTURA, 2015).

Apesar da expressiva diminuição do número de casos de poliomielite, cerca de 12 milhões de pessoas convivem com as consequências da doença (FARIA, 2015). A fraqueza muscular, um dos principais sintomas da SPP, acarreta uma sobrecarga energética durante as atividades rotineiras, gerando fadiga excessiva que associada à dor mioarticular compromete a capacidade funcional desses indivíduos e a qualidade de vida (SOARES et al., 2019).

Nesse sentido, considerando ser uma doença crônica e incurável, compreende-se que os pacientes necessitam da atenção de uma equipe multidisciplinar, sendo essencial que a fisioterapia esteja inserida nesse cuidado, a fim de contribuir para a melhora da função respiratória, da mobilidade física, redução da dor e, consequentemente, na melhora da qualidade de vida dos indivíduos afetados (GARIP et al., 2017).

Em função disso, ou seja, do aumento de números de casos de SPP na última década e da escassez de estudos que reportem acerca do tratamento 
fisioterapêutico realizado em grupo para a melhora da qualidade de vida e capacidade funcional de indivíduos com a SPP, justifica-se a importância da realização de estudos interventivos, a exemplo deste, a fim de consolidar evidências científicas sobre o tema em questão.

Ante ao exposto, esta pesquisa parte da hipótese de que o atendimento fisioterapêutico, em grupo, é uma estratégia eficaz para a melhora da dor, qualidade de vida e capacidade funcional de indivíduos com a SPP. Para tanto, emergiu a seguinte questão problematizadora: o atendimento fisioterapêutico, realizado em grupo, constitui-se como uma estratégia importante para melhorar a dor, capacidade funcional e a qualidade de vida de indivíduos com a SPP?

$\mathrm{Na}$ expectativa em se obter dados confiáveis sobre a temática e responder ao questionamento supracitado o presente estudo teve como objetivo analisar o impacto do tratamento fisioterapêutico em grupo na melhora da dor, capacidade funcional e qualidade de vida de indivíduos com a SPP.

\section{Metodologia}

Pesquisa transversal de caráter exploratória-descritiva com abordagem quantitativa desenvolvida na Fundação Centro Integrado de Apoio ao Portador de Deficiência (FUNAD) na cidade de João Pessoa-Paraíba. A escolha do cenário justificou-se devido a instituição ser referência em atendimento multidisciplinar a pessoas com deficiência física e intelectual, dentre os quais pacientes com a SPP.

Dos 12 existentes participaram da pesquisa 10 pacientes diagnosticados com a SPP que realizavam, desde 2017, tratamento fisioterapêutico, em grupo, por alunos do $7^{\circ}$ período do curso de fisioterapia da Universidade Federal da Paraíba (UFPB) sob supervisão docente. Foram incluídos àqueles pacientes que frequentavam o grupo de fisioterapia há pelo menos um ano e excluídos àqueles que tenham se ausentado do grupo por três sessões sequenciais sem justificativa; e os que se recusaram a participar da pesquisa.

Ao ser admitido no grupo de atendimento fisioterapêutico para pacientes com a SPP, cada participante foi submetido a uma avaliação neurofuncional antes de iniciar o tratamento em grupo. As sessões foram realizadas duas vezes por semana, totalizando 10 sessões mensais, com duração média de 60 
minutos cada. As intervenções fisioterapêuticas envolveram a realização de alongamentos ativos e passivos da musculatura do tronco, membros superiores e inferiores para ganho e/ou manutenção da amplitude de movimento; exercícios isométricos, ativos, passivos e ativo assistidos para manutenção da força muscular de membros superiores, inferiores e tronco; atividades lúdicas que estimulem a funcionalidade; e exercícios de equilíbrio, propriocepção e mobilidade. Além disso, realizaram-se massagens, relaxamentos musculares, dinâmicas em grupo e discussões sobre as dúvidas relacionadas a doença e outros aspectos de saúde, conforme a necessidade do grupo.

Todas as atividades desenvolvidas nas sessões em grupo foram atreladas ao uso da musicoterapia e planejadas pelos discentes e docente responsável, buscando-se respeitar e atender as necessidades individuais num contexto coletivo.

A coleta de dados ocorreu, nos turnos matutino e vespertino (13:00 às 17:00 horas), no mês de fevereiro de 2020, por meio de entrevista e avaliação fisioterapêutica individual norteada por instrumentos estruturados que contemplaram a mensuação das variáveis dor, por meio Escala Visual Analógica da Dor (EVA); qualidade de vida com o WHOQOL-bref; e a capacidade funcional por meio da Escala de Atividades de Vida Diária de Barthel, para fins de comparação com os escores iniciais obtidos a partir dos registros em ficha de avaliação neurofuncional individual utilizada na disciplina "Fisioterapia Aplicada nas Disfunções do Sistema Nervoso" durante a admissão do participante no grupo de fisioterapia. As entrevistas ocorreram com duração média de 20 minutos

Para as análises estatísticas quantitativas, utilizou-se o programa Microsoft Excel versão 2016. Foi realizada a análise descritiva das variáveis pesquisadas, cujos dados foram apresentados sob forma de tabelas por distribuição de frequência relativa e percentual, sendo os resultados discutidos à luz da literatura pertinente.

O estudo foi aprovado pelo comitê de Ética em Pesquisa do Centro de Ciências da Saúde da Universidade Federal da Paraíba sob o parecer no 4.003.241 com CAAE: 30001920.5.0000.5188), conforme exigências 
estabelecidas pela Resolução 466/12 do Conselho Nacional de Saúde que norteia a prática de pesquisa com seres humanos (BRASIL, 2012).

\section{Resultados}

Participaram 10 pacientes, com idade entre 37 e 58 anos, baixa condição socioeconômica, união conjugal estável, oito ou mais anos de estudo e renda familiar mensal de um a três salários mínimos.

Constatou-se, conforme Tabela 1, a redução do quadro álgico dos pacientes após admissão no grupo para realização das sessões fisioterapêuticas convencionais comparado a pré intervenção. A predominância dos pacientes apresentava escores de dor entre 8 a $10(90,0 \%)$ na EVA caracterizada como intensa previamente ao início das sessões fisioterapêuticas. Após o início das intervenções fisioterapêuticas em grupo, nota-se a diminuição da percepção da dor para grau leve $(80,0 \%)$ e moderada $(20,0 \%)$, com escores variando de 0 a 3 , conforme a autoavaliação dos pacientes.

Tabela 1 - Análise comparativa da dor pela Escala Analógica Visual da Dor antes e após o tratamento fisioterapêutico em grupo para pacientes com a SPP, João Pessoa - Paraíba, Brasil, 2020.

\begin{tabular}{l|cc|cc}
\hline \multicolumn{1}{c|}{ VARIÁVEIS } & \multicolumn{2}{c}{ ANTES } & \multicolumn{2}{c}{ APÓS } \\
\hline Pacientes & Escore & Classificação & Escore & Classificação \\
\hline P1 & 9 & DI & 2 & DL \\
P2 & 8 & DI & 2 & DL \\
P3 & 10 & DI & 3 & DM \\
P4 & 10 & DI & 3 & DM \\
P5 & 9 & DI & 1 & DL \\
P6 & 8 & DM & 2 & DL \\
P7 & 8 & DI & 2 & DL \\
P8 & 8 & DI & 1 & DL \\
P9 & 9 & DM & 2 & DL \\
P10 & 7 & DM & 2 & DL \\
\hline Nota: ${ }^{*}=$ Paciente; ${ }^{* *}$ DI=Dor Intensa; ${ }^{* * *}$ DM=Dor Moderada; ${ }^{* * * * D L=D o r ~ L e v e ~}$ &
\end{tabular}

$\mathrm{Na}$ análise comparativa da mobilidade por meio do Índice de Barthel, que avaliou as atividades da vida diária e a independência funcional no cuidado pessoal, locomoção e eliminações fisiológicas dos pacientes com SPP, constatou-se melhora dos escores quanto o nível de dependência para todos 
os pacientes após o início do tratamento fisioterapêutico em grupo quando comparado ao período pré-intervenção, conforme evidenciado na Tabela 2.

Tabela 2 - Análise comparativa da capacidade funcional pelo Índice de Barthel antes e após tratamento fisioterapêutico em grupo para pacientes com a SPP, João Pessoa - Paraíba, Brasil, 2020.

\begin{tabular}{l|c|c|c|c}
\hline \multicolumn{1}{|c|}{ VARIÁVEIS } & \multicolumn{2}{|c|}{ ANTES } & \multicolumn{2}{c}{ APÓS } \\
\hline \multicolumn{1}{c}{ Pacientes } & Escore & Classificação & Escore & Classificação \\
\hline P1 & 61 & $\mathrm{DM}$ & 99 & $\mathrm{DL}$ \\
P2 & 62 & $\mathrm{DM}$ & 91 & $\mathrm{DL}$ \\
P3 & 65 & $\mathrm{DM}$ & 80 & $\mathrm{DL}$ \\
P4 & 50 & $\mathrm{DM}$ & 82 & $\mathrm{DL}$ \\
P5 & 91 & $\mathrm{DL}$ & 100 & $\mathrm{I}$ \\
P6 & 92 & $\mathrm{DL}$ & 99 & $\mathrm{DL}$ \\
P7 & 91 & $\mathrm{DL}$ & 99 & $\mathrm{DL}$ \\
P8 & 90 & $\mathrm{DL}$ & 98 & $\mathrm{DL}$ \\
P9 & 61 & $\mathrm{DM}$ & 99 & $\mathrm{DL}$ \\
P10 & 91 & $\mathrm{DL}$ & 100 & $\mathrm{I}$ \\
\hline Nota: ${ }^{*}=$ Paciente; ${ }^{* *}$ DM=Dependência Moderada; & F* DL=Dependência Leve; ${ }^{* * *} \mid=$ Independência. \\
\end{tabular}

Sobre a avaliação quantitativa da qualidade de vida com o WHOQOLbref, a Tabela 3 mostra a descrição dos escores segundo resultados das análises comparativas antes e após a intervenção fisioterapêutica em grupo. Observou-se a melhora da qualidade de vida para todos os domínios analisados.

Os Gráficos 1 e 2 apresentam, respectivamente, os resultados dos domínios do questionário WHOQOL-bref sobre a qualidade de vida dos pacientes de SPP antes e após a realização do tratamento fisioterapêutico em grupo. Os dados revelam uma melhora da qualidade de vida em todos os domínios, sendo o físico, psicológico e de relações sociais os que mais expressaram variação positiva.

\section{Discussão}

A SPP se caracteriza por sintomas musculoesqueléticos como a presença de fadiga, dor, redução de massa e força muscular, flexibilidade articular diminuída, déficit para locomoção e alterações posturais (ALMEIDA et al., 2016). A cronicidade dos sintomas na SPP, a exemplo da dor, pode resultar em importante incapacidade física e emocional do indivíduo, gerando impacto negativo sobre o seu bem-estar e qualidade de vida (FARIA, 2015). 
Tabela 3 - Análise comparativa da qualidade de vida com o WHOQOL-bref antes e após tratamento fisioterapêutico em grupo para pacientes com a SPP, João Pessoa - Paraíba, Brasil, 2020.

\begin{tabular}{|c|c|c|}
\hline DOMÍNIOS & ANTES & APÓS \\
\hline \multicolumn{3}{|l|}{ Físico } \\
\hline Média $\pm D P$ & $8,00 \pm 0,93$ & $16,46 \pm 1,37$ \\
\hline Valor máximo & 9,71 & 18,86 \\
\hline Valor mínimo & 6,86 & 14,86 \\
\hline Amplitude & 2,86 & 4,00 \\
\hline Coeficiente de variação & 11,66 & 8,31 \\
\hline \multicolumn{3}{|l|}{ Psicológico } \\
\hline Média $\pm D P$ & $9,33 \pm 1,09$ & $17,33 \pm 1,18$ \\
\hline Valor máximo & 11,33 & 18,67 \\
\hline Valor mínimo & 8,00 & 15,33 \\
\hline Amplitude & 3,33 & 3,33 \\
\hline Coeficiente de variação & 11,66 & 6,78 \\
\hline \multicolumn{3}{|l|}{ Relações sociais } \\
\hline Média $\pm D P$ & $8,67 \pm 2,37$ & $16,80 \pm 1,80$ \\
\hline Valor máximo & 12,00 & 20,00 \\
\hline Valor mínimo & 5,33 & 14,67 \\
\hline Amplitude & 6,67 & 5,33 \\
\hline Coeficiente de variação & 27,38 & 10,71 \\
\hline \multicolumn{3}{|l|}{ Meio ambiente } \\
\hline Média $\pm D P$ & $12,55 \pm 0,93$ & $14,20 \pm 1,06$ \\
\hline Valor máximo & 14,00 & 15,50 \\
\hline Valor mínimo & 11,00 & 12,50 \\
\hline Amplitude & 3,00 & 3,00 \\
\hline Coeficiente de variação & 7,38 & 7,46 \\
\hline \multicolumn{3}{|l|}{ Auto-avaliação da QV } \\
\hline Média $\pm D P$ & $6,40 \pm 1,84$ & $16,60 \pm 1,35$ \\
\hline Valor máximo & 10,00 & 18,00 \\
\hline Valor mínimo & 4,00 & 14,00 \\
\hline Amplitude & 6,00 & 4,00 \\
\hline Coeficiente de variação & 28,72 & 8,13 \\
\hline \multicolumn{3}{|l|}{ TOTAL } \\
\hline Média土DP & $9,66 \pm 0,57$ & $16,02 \pm 0,80$ \\
\hline Valor máximo & 10,46 & 17,38 \\
\hline Valor mínimo & 8,92 & 14,92 \\
\hline Amplitude & 1,54 & 2,46 \\
\hline Coeficiente de variação & 5,95 & 4,97 \\
\hline
\end{tabular}




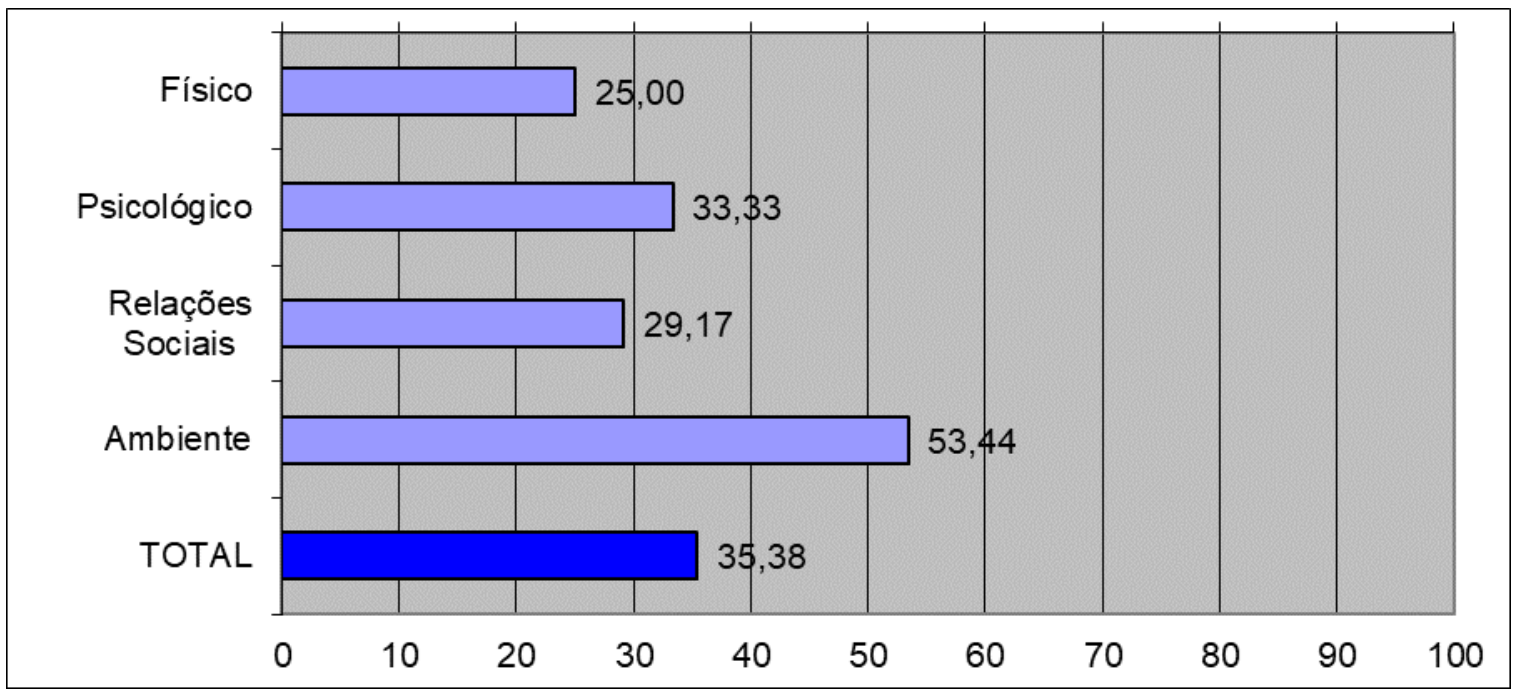

Gráfico 1 - Variações dos domínios do Índice WHOQOL-bref, permutados em valores de percentuais dos pacientes com a SPP antes o tratamento fisioterapêutico. João Pessoa, Paraíba, Brasil. 2020.

Fonte: Dados da pesquisa (2020).

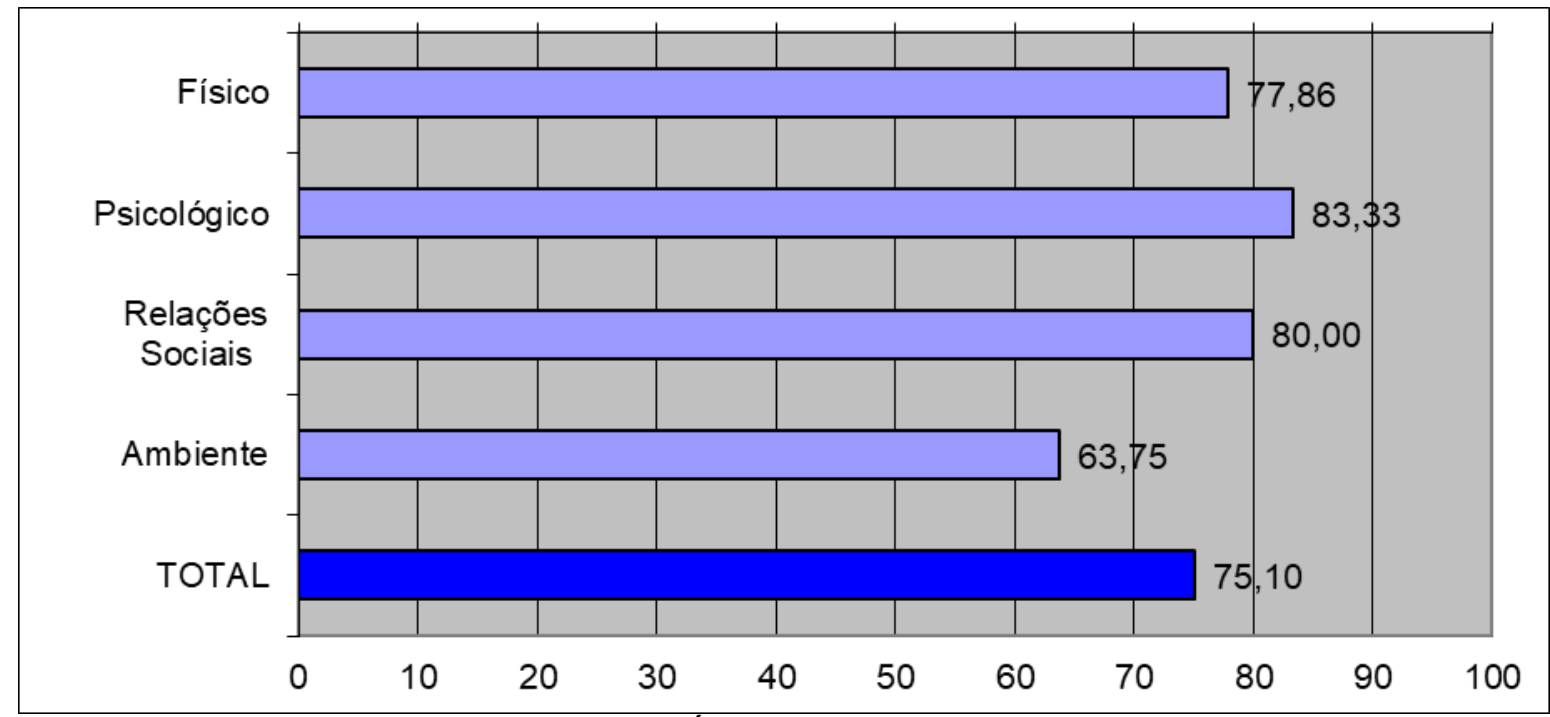

Gráfico 2 - Variações dos domínios do Índice WHOQOL-bref, permutados em valores de percentuais dos pacientes com a SPP após o tratamento fisioterapêutico. João Pessoa, Paraíba, Brasil. 2020.

Fonte: Dados da pesquisa (2020).

A sensação de dor consiste em experiência complexa, no qual muitas vezes, difícil de ser mensurada devido à ausência de marcador biológico específico. Entretanto, o autorregistro e a descrição individual, conforme obtido dos participantes durante as avaliações, geralmente fornecem evidências acuradas, fidedignas e suficientes para detectar a presença e a intensidade da dor (CASTRO; PEREIRA; BASTOS, 2018). 
Dessa forma, o uso da escala EVA para mensuração da dor pré e pós intervenção possibilitou que cada participante pudesse apontar, mediante 0 contato visual, o grau de sua dor. Os registros periódicos de sua intensidade são considerados fundamentais para o acompanhamento da evolução dos pacientes, realização de ajustes e avaliação da eficácia do tratamento implementado (HEINEN et al., 2016). No caso da pesquisa atual, a implementação da cinesioterapia motora convencional atrelada à massoterapia e aos exercícios de relaxamento foram elencados como principais estratégias para os indivíduos com SPP.

Estar inserido no grupo para a realização do tratamento fisioterapêutico pautado na cinesioterapia motora revelou-se uma condição eficaz para a redução da dor dos indivíduos com a SPP, condizendo as evidencias clínicas de que nos casos de dor crônica o exercício físico é considerado necessário para atenuar as instabilidades musculoesqueléticas e obter resultados satisfatórios daqueles indivíduos com sintomas álgicos exacerbados (ORSINI et al., 2011).

$\mathrm{Na}$ SPP, é frequente a ocorrência de encurtamentos musculares, contraturas articulares, déficit de força e alterações posturais (ALMEIDA et al., 2016; ORSINI et al., 2010). Nesse sentido, a implementação de alongamento, exercícios isotônicos, isométricos e isocinéticos em limites submáximos são fundamentais para o recrutamento muscular sem gerar sobrecarga e fadiga muscular (ORSINI et al., 2011).

O exercício físico, sobretudo a cinesioterapia, promove respostas motoras, autonômicas, neuroendócrinas, emocionais, comportamentais e de percepção corporal, reguladas pelo sistema límbico, impactando diretamente nos sintomas psicossomáticos (SCHNORNBERGER; JORGE; WIBELINGER, 2017), conforme evidenciado nos indivíduos com SPP, em que houve uma redução significativa da dor categorizada na EVA como intensa previamente aos exercícios para um nível leve a moderado pós intervenção fisioterapêutica em grupo.

Assim como no estudo desenvolvido em São Paulo para pacientes com doença crônica em que a cinesioterapia se mostrou eficaz na redução da dor e fadiga muscular (SOUSA et al., 2018), os resultados da pesquisa atual refletem 
que a proposta de tratamento fisioterapêutico desenvolvida em grupo por meio de exercícios isométricos, ativos e passivos, alongamentos musculares, massoterapia e relaxamentos também exerceu influência clínica positiva junto aos indivíduos com SPP.

Para autores, a justificativa para a redução da dor está na liberação de endorfinas e ativação de vias descendentes inibitórias endógenas da dor durante a prática dos exercícios (OLIVEIRA; SILVA, 2017). Ademais, a dor neuropática, presente em lesões parciais ou totais das vias nervosas do sistema nervoso periférico, como na SPP, possibilita a melhor resposta analgésica mediante interferência na geração do impulso nociceptivo, nos processos de transmissão e de interpretação do fenômeno doloroso (ARAÚJO; ROMERO, 2015).

Além da redução do quadro álgico e da fadiga muscular, o tratamento fisioterapêutico também se mostrou eficaz na melhora da funcionalidade dos participantes evidenciada pela mudança no nível de dependência de moderado para leve, corroborando pesquisa de intervenção com pacientes neurológicos em que a fisioterapia desenvolvida em grupo revelou aumento nos escores do equilíbrio pré e pós-intervenção, a partir da escala de Berg (AMARAL-FELIPE et al., 2016).

A funcionalidade refere-se à capacidade em realizar as atividades de vida diária de maneira independente, estando diretamente associada à preservação do equilíbrio físico, visto que, as efetivações das atividades exigem do indivíduo a manutenção da postura ereta (AGUIAR et al., 2019). Contudo, em situações adversas como nas doenças crônicas, processos traumáticos e cirúrgicos há comprometimento da autonomia e da independência funcional (WIETHAN; SOARES; SOUZA, 2017).

Desse modo, os exercícios terapêuticos realizados regularmente, além de promover o fortalecimento muscular possibilitam a reorganização cerebral com reflexo na otimização do desempenho funcional para a realização das atividades cotidianas (THORNBERG; JOSEPHSSON; LINDQUIST, 2014). Esse aspecto pôde ser observado a partir dos escores obtidos no Índice de Barthel pós-intervenção, os quais revelaram melhora da independência 
funcional no cuidado pessoal, locomoção e eliminações fisiológicas dos pacientes com SPP quando comparado ao período de pré-intervenção.

A realização das atividades em grupo além de ter possibilitado a melhora dos sintomas físicos também proveu a melhora de sintomas emocionais e maior socialização entre os membros do grupo com SPP, conforme evidenciado nos domínios de qualidade de vida. Conforme estudo, a atividade em grupo é uma das possibilidades da terapêutica que pode fortalecer amizades, minimizar sentimentos de solidão e rejeição (CARVALHO et al., 2014).

As trocas de experiências entre pessoas que vivenciam situações e problemas de saúde semelhantes podem desenvolver resultados benéficos para a reabilitação, sendo consideradas, muitas vezes, cruciais para a mudança de comportamento e cura física e mental (RECCO; LOPES, 2016). Nesse caso, a realização da fisioterapia em grupo mostrou-se um recurso importante para os indivíduos com SPP, visto que, possibilitou atenuação de sintomas físicos impactando diretamente na qualidade de vida dos participantes.

O conceito qualidade de vida tem sido foco de muitos estudos nas últimas décadas, nas mais diferentes áreas do conhecimento, sendo definida pela Organização Mundial da Saúde como a percepção que o indivíduo tem de sua própria condição de vida, dentro do seu próprio contexto de cultura e sistema de valores, considerando seus objetivos de vida, as expectativas e as preocupações (CRUZ; COLLET; NÓBREGA, 2018).

Neste estudo, assim como as variáveis: dor e capacidade funcional buscou-se avaliar a qualidade de vida dos indivíduos a partir da comparação dos domínios do WHOQOL-bref antes e após a intervenção fisioterapêutica.

Observou-se uma melhora significativa na qualidade de vida global após admissão dos indivíduos no serviço de fisioterapia para a realização dos exercícios em grupo. Essa evolução pôde ser evidenciada nos resultados dos domínios físico, psicológico e relações sociais, os quais apresentaram pontuações elevadas na avaliação pós-intervenção, sendo o domínio meio ambiente o que menos contribuiu para a qualidade de vida na análise comparativa. 
Dessa maneira, os resultados apontam que a inserção da fisioterapia na rotina semanal foi uma medida benéfica para a melhora das queixas físicas e emocionais apresentadas pelos participantes anteriormente à admissão ao grupo.

Apesar das evidencias plausíveis apresentadas nesse estudo de que a fisioterapia em grupo propicia benefícios importantes para a saúde física e mental de indivíduos com SPP, é necessário apontar limitações do estudo, sendo elas: amostra reduzida; e ausência de um grupo controle a fim de comparar os resultados obtidos entre os grupos para maior evidência científica.

\section{Conclusão}

Ao término da pesquisa constatou-se que os atendimentos fisioterapêuticos em grupo, por meio da cinesioterapia motora convencional, constituíram-se como uma estratégia efetiva na melhora da dor, capacidade funcional e qualidade de vida de indivíduos com a SPP.

A efetividade da fisioterapia grupal pôde ser constatada por meio da comparação dos resultados quantitativos obtidos a partir da aplicação dos instrumentos de avaliação para as variáveis supracitadas pré e pósintervenção.

Observou-se que além dos benefícios físicos, a fisioterapia em grupo possibilitou uma melhora da qualidade de vida global dos participantes quanto aos domínios relações sociais e psicoemocional.

A pesquisa proporcionou uma oportunidade singular de avaliar e compreender as repercussões que a fisioterapia pode ocasionar nas pessoas com SPP e que a prática fisioterapêutica realizada em grupo pode ser uma medida fundamental para o bem-estar físico e psíquico de pacientes com doença crônica SPP.

Apesar dos significativos resultados da pesquisa, sugerimos que novos estudos sejam realizados buscando comparar a prática da cinesioterapia individualizada e de forma coletiva, afim de conhecer quais os efeitos e, para qual tipo de paciente possui melhor eficácia. 
Ademais, entendemos que são necessários mais estudos sobre a temática, a fim de enriquecer o meio científico sobre a SPP cujas pesquisas ainda são escassas.

\section{Referências}

AGUIAR, V. F. F. et al. Avaliação da capacidade funcional e qualidade de vida do idoso no Brasil residente em comunidade. Revista de Enfermagem Referência, Coimbra, v. 4, n. 21, p. 59-66, jun./2019. Disponível em: http://www.scielo.mec.pt/scielo.php?script=sci_arttext\&pid=S087402832019000200006. Acesso em: 8 jul. 2020.

ALMEIDA, C. R. D. S. et al. Percepção dos acadêmicos de fisioterapia sobre a síndrome pós-poliomielite. Temas em Saúde, Paraíba, ed. especial, p. 516-529, 2016. Disponível em: http://temasemsaude.com/edicao-especial-conesf/. Acesso em: 13 jul. 2020.

AMARAL-FELIPE, K. M. et al. Fisioterapia em grupo melhora o equilíbrio e a funcionalidade de indivíduos com hemiparesia. Conscientiae Saúde, São Paulo, v. 15, n. 3, p. 385-391, ago./2016. Disponível em: https://pesquisa.bvsalud.org/portal/resource/pt/biblio-846622. Acesso em: 1 jul. 2020.

ARAUJO, L. C. D; ROMERO, B. Pain: evaluation of the fifth vital sign. A theoretical reflection. Revista Dor, São Paulo, v. 16, n. 4, p. 291-296, out./2015. Disponível em: https://www.scielo.br/scielo.php?pid=S1806-00132015000400291\&script=sci_abstract. Acesso em: 13 jul. 2020.

BRASIL, MINISTÉRIO DA SAÚDE. Situação epidemiológica - dados, 2018. Disponível em: https://saude.gov.br/o-ministro/965-saude-de-a-a-z/poliomieliteparalisia-flacida-aguda/11425-situacao-epidemiologica-dados. Acesso em: 11 dez. 2019.

BRASIL. Resolução ํo 466, de 12 de dezembro de 2012. Dispõe sobre diretrizes e normas regulamentadoras de pesquisas envolvendo seres humanos. Diário Oficial [da] República Federativa do Brasil, Brasília, DF, 13 jun. 2013. Disponível em: <Disponível em: http://bit.ly/1mTMIS3 > Acesso em: 16 nov. 2020.

CARVALHO, A. C. et al. Fisioterapia em Grupo: um modelo terapêutico para pacientes com doença de Parkinson - relato de experiência. Revista Adapta, Presidente Prudente, v. 10, n. 1, p. 11-16, Jan./Dez., 2014.

CASTRO, C. C. D; PEREIRA, A. K. D. S; BASTOS, B. R. Implementação da avaliação da dor como o quinto sinal vital. Revista de Enfermagem UFPE online, v. 12, n. 11, p. 3009-3014, nov./2018. Disponível em: https://pesquisa.bvsalud.org/portal/resource/pt/biblio-997579. Acesso em: 5 jul. 2020.

CRUZ, D. S. M. D; COLLET, N.; NÓBREGA, V. M. Qualidade de vida relacionada à saúde de adolescentes com dm1- revisão integrativa. Ciência e saúde coletiva, Rio de Janeiro, v. 23, n. 3, p. 973-989, mar./2018. Disponível em: https://www.scielo.br/scielo.php?script=sci_arttext\&pid=S1413-81232018000300973.

Acesso em: 12 jul. 2020. 
DURANTE, A. L. T. D. C; POZ, M. R. D. Saúde global e responsabilidade sanitária brasileira: o caso da erradicação da poliomielite. Saúde em Debate, Rio de Janeiro, v. 38 , n. 100, p. 129-138, jan./2014. Disponível em: https://www.scielo.br/scielo.php?pid=S0103-

11042014000100129\&script=sci_abstract\&tIng=pt. Acesso em: 10 dez. 2019.

FARIA, L. Poliomielite: várias histórias da doença e de seus efeitos tardios. História, Ciências, Saúde-Manguinhos, Rio de Janeiro, v. 22, n. 3, p. 1081-1087, jul./2015. Disponível em: https://www.scielo.br/scielo.php?pid=S010459702015000301081\&script=sci_arttext. Acesso em: 4 dez. 2019.

GARIP, Y. et al. O. Qualidade de vida relacionada com a saúde em sobreviventes turcos da pólio: impacto pós- -pólio na saúde relacionada com a qualidade de vida em termos de estado funcional, gravidade da dor, fadiga e funcionamento social e emocional. Rev Bras Reumatol [Internet]. v. 57 n. 1, p. 1-7, 2017. Disponível em: http://www.scielo.br/pdf/rbr/v57n1/0482-5004-rbr-57-01-0001. Pdf

HEINEN, A. C. et al. Avaliação da dor como quinto sinal vital: uma escolha profissional de intervenção fisioterapêutica. Revista Pesquisa em Fisioterapia, v. 6, n. 4, p. 379386, nov./2016. Disponível em: https://www5.bahiana.edu.br/index.php/fisioterapia/article/view/935. Acesso em: 13 jul. 2020.

OLIVEIRA, A. S.; SILVA, J. G. Efeito de um programa de tratamento fisioterapêutico em paciente com poliartralgia persistente pós-febre de chikungunya. Relato de caso. Rev. dor, São Paulo , v. 18, n. 4, p. 370-373, dez. 2017. Disponível em: $<$ http://www.scielo.br/scielo.php?script=sci_arttext\&pid=S1806-

00132017000400370\&lng=en\&nrm=iso $>$. Acesso em: 25 Set. 2020. https://doi.org/10.5935/1806-0013.20170132.

OPAS - Organização Pan Americana de Saúde. Folha informativa - Poliomielite. Disponível em: https://www.paho.org/bra/index.php?option=com_content\&view=article\&id=5735:folhainformativa-poliomielite\&ltemid=820. Acesso em: 10 dez. 2019.

ORSINI, M. et al. Gerenciamento da dor na Síndrome Pós-poliomielite: estudo de caso. Fisioterapia e Pesquisa, v. 18, n. 4, p. 382-387, out./2011. Disponível em:https://www.scielo.br/scielo.php?pid=S1809-

29502011000400015\&script=sci_abstract\&tlng=pt. Acesso em: 12 jul. 2020.

ORSINI, M. et al. Guia de Reabilitação Neurológica na Síndrome Pós-Poliomielite: abordagem interdisciplinar. Revista Neurociências, v. 18, n. 2, p. 204-213, 2010. Disponível em: https://periodicos.unifesp.br/index.php/neurociencias/article/view/8483. Acesso em: 6 jul. 2020.

RECCO, R. A. C; LOPES, S. M. B. Sobre fisioterapia e seus recursos terapêuticos: o grupo como estratégia complementar à reabilitação. Trabalho, Educação e Saúde, Rio de Janeiro, v. 14, n. 2, p. 593-610, ago./2016. Disponível em: https://www.scielo.br/scielo.php?script=sci_arttext\&pid=S1981-

$77462016000200593 \&$ Ing=en\&nrm=iso\&tlng=pt. Acesso em: 9 jul. 2020

RECHENCHOSKI, D. Z. et al. Poliomielite - erradicação ou controle?. Revista de

Ciências Médicas e Biológicas, Salvador, v. 14, n. 2, p. 233-237, mai./2015. 
Disponível em: https://portalseer.ufba.br/index.php/cmbio/article/view/13413. Acesso em: 10 dez. 2019.

SCHNORNBERGER, C. D. M; JORGE, M. S. G; WIBELINGER, L. M. Physiotherapeutic intervention in pain and quality of life of women with rheumatoid arthritis. Case reports. Revista Dor, São Paulo, v. 18, n. 4, p. 365-369, dez./2017. Disponível em: https://www.scielo.br/scielo.php?script=sci_arttext\&pid=S180600132017000400365 . Acesso em: 16 jun. 2020.

SOARES, N. S. C. S. et al. Conhecimentos dos enfermeiros de unidades básicas de saúde sobre a síndrome pós-poliomielite. Enfermagem em foco, Brasília, v. 10, n. 1, p. 52-57, 2019. Disponível em: https://pesquisa.bvsalud.org/portal/resource/pt/bde34575. Acesso em: 1 fev. 2020.

SOUSA, B. S. M. et al. O efeito da cinesioterapia e hidrocinesioterapia sobre a dor, capacidade funcional e fadiga em mulheres com fibromialgia. Conscientiae Saúde, v. 17, n. 3, p. 231-238, 2018. Disponível em: https://periodicos.uninove. br/index.php?journal=saude \&page=article\&op=view\&path\%5 $\mathrm{B} \% 5 \mathrm{D}=8010$. Acesso em: 9 jul. 2020.

TAVARES, F. N. O início do fim da poliomielite: 60 anos do desenvolvimento da vacina. Revista Pan-Amazônica de Saúde, v. 6, n. 3, p. 9-11, set./2015. Disponível em: $\quad$ http://scielo.iec.gov.br/scielo.php?script=sci_arttext\&pid=S217662232015000300001. Acesso em: 3 fev. 2020.

THORNBERG, K.; JOSEPHSSON, S.; LINDQUIST, I. Experiences of participation in rhythm and movement therapy after stroke. Disability and Rehabilitation, v. 36, n. 22, p. 1869 1874, 2014. Doi:10.3109/09638288.2013.876107

VENTURA, T. Poliomielite e seus efeitos tardios: viver ao ritmo da doença. Rev Port Med Geral Fam, v. 31, p. 326-333, out./2015. Disponível em: http://www.scielo.mec.pt/pdf/rpmgf/v31n5/v31n5a06.pdf. Acesso em: 17 jun. 2020.

WIETHAN, J. R. V.; SOARES, J. C.; SOUZA, J. A. Avaliação da funcionalidade e qualidade de vida em pacientes críticos: série de casos. Revista Acta Fisiátrica, v. 24, n. 1, p. 7-12, 2017. Disponível em: https://pesquisa.bvsalud.org/portal/resource/pt/biblio-906641 ?lang=pt. Acesso em: 25 set. 2020. 